\title{
Проблемы тестирования V2X и их решения
}

\author{
М. Соковишин ${ }^{1}$, С. Литвиненко
}

УДК 621.317.7.023:[621.396.931:004.72] | ВАК 05.11.01

\begin{abstract}
В настоящее время автомобильная отрасль - одна из тех областей промышленности, в которых технологии развиваются особенно быстро. Это, в частности, проявляется в появлении огромного количества инноваций, связанных с беспилотным транспортом. Хотя автономные автомобили пока остаются в будущем, это будущее уже не так далеко, как казалось всего несколько лет назад. Автономное вождение предполагает постоянное "общение" автомобиля с другими транспортными средствами, транспортной инфраструктурой и прочими объектами. Системы коммуникации беспилотных автомобилей с внешней средой обладают рядом особенностей, которые будут предъявлять новые требования к контрольно-измерительному и тестовому оборудованию. В данной статье, основанной на презентации, представленной M. Соковишиным на семинаре компании Keysight Technologies "Основы тестирования автомобильных цифровых и беспроводных устройств", который прошел 28 мая 2019 года в Москве, приводятся общие сведения о текущем положении в развитии и стандартизации таких систем, а также о некоторых решениях для их тестирования, которые уже сейчас предлагает компания Keysight.
\end{abstract}

еспилотные автомобили, очевидно, потребуют наличия беспроводных каналов связи с внешней средой. Однако такая коммуникация может не только служить целям автономного вождения, но и оказывать помощь водителю, а также способствовать повышению безопасности движения обычных транспортных средств. Например, автомобиль может получать в реальном времени данные о свободных местах на парковке в месте назначения, передавать информацию о своем техническом состоянии в сервисные центры. Он может сообщать о своих координатах в центры управления трафиком и получать от них рекомендации о предпочтительном маршруте движения с учетом пробок, а центры на основе данных от большого количества автомобилей могут регулировать, например, режимы работы светофоров, более равномерно распределяя загруженность дорог в городе. Автомобиль может устанавливать связь с другими транспортными средствами и даже со смартфонами, лежащими в карманах пешеходов, благодаря этому предупреждая аварийные ситуации. Преимуществ у таких систем множество.

Поскольку с помощью такой системы автомобиль может коммуницировать с различными объектами, эти системы называют V2X, что расшифровывается как

ООО «Кейсайт Текнолоджиз», инженер технической поддержки.
Vehicle-to-X, или "Транспортное средство - X». Здесь X переменная, на месте которой могут быть разные объекты. Сейчас в рамках этого термина различают следующие основные виды коммуникации:

- V2V (Vehicle-to-Vehicle) - связь автомобиля с другим транспортным средством, прежде всего, для предотвращения аварийных ситуаций, а также для получения информации о движении, препятствияхит. п.;

- V2I (Vehicle-to-Infrastructure) - связь с транспортной инфраструктурой, обеспечивающая, например, получение предупреждений о смене сигнала светофора, пересечении улицы пешеходами, состоянии дорожного покрытия и т. п.;

- V2N (Vehicle-to-Network) - связь с сетью для получения доступа к различной информации (например, о трафике), вызова экстренных служб, получения различныхуслуг, доступа к облачным ресурсам;

- V2P (Vehicle-to-Pedestrian) - связь с пешеходом, направленная на повышение безопасности.

В настоящее время консорциумом 3GРP разрабатывается релиз 16 стандарта 3GРP, в котором будет стандартизирована беспроводная связь автомобиля с внешней средой в сетях 5С. В данном стандарте она называется C-V2X. Буква С здесь означает Cellular - "сотовый», отражая то, что в основе этого стандарта - сотовая сеть. 
Однако C-V2X - не единственный стандарт, претендующий на ведущую роль в обеспечении коммуникации автомобиля с внешней средой. Его конкурент - DSRC, или 802.11р, основанный на стандарте Wi-Fi - включен в ряд принятых стандартов, таких как американские SAE 12735 и SAE 22945, европейский ITS G5 и японский ARIB STD-109, и уже используется для определенных задач преимущественно в США, например для внесения платы за использование дорог.

Оба стандарта обладают своими преимуществами и недостатками.

Главное преимущество стандарта DSRC заключается в его готовности с точки зрения стандартизации. Кроме того, будучи основанным на стандарте Wi-Fi, он работает на частотах около 5, 9 ГГц (за исключением Японии, где используется частотный диапазон 760 МГц), которые менее загружены, чем диапазоны сотовой связи. В стандарте применяется выделенный канал, что обеспечивает низкую задержку - менее 5 мс. Вместе с тем дальность действия связи DSRC относительно небольшая, что требует создания сети с очень большим количеством точек доступа. Если при этом учесть, что модель монетизации услуг сети DSRC не вполне ясна, сложно сказать, каким образом предполагается окупить создание такой сети.

Стандарт C-V2X лишен этих недостатков: для его применения, вообще говоря, не требуется развертывания специальной сети, поскольку он основан на использовании инфраструктуры сотовой связи, а проблема монетизации может быть относительно легко решена с помощью специальных тарифов сотовых операторов.

Еще одно преимущество стандарта C-V2X перед DSRC его бо́льшая защищенность, определяющаяся средствами шифрования сотовой сети. Это очень важный аспект в коммуникациях между автомобилем и внешней средой, поскольку они предполагают не только передачу конфиденциальной информации, но и обмен данными, впрямую влияющими на безопасность движения.

Помимо коммуникации через сотовую сеть по протоколу, получившему название Uu, в C-V2X предусмотрена и возможность прямого "общения" между транспортными средствами. Это обеспечивается службой Proximity Service (ProSe) с использованием интерфейса РС5 (рис. 1). Дальность действия данной связи составляет около километра. Это, помимо прочего, обеспечивает определенную независимость коммуникационной системы от доступности сети сотовой связи. В частности, недостатками существующих в настоящее время систем экстренного вызова, таких как "ЭРА-ГЛОНАСС" и европейская система eCall, является то, что если авария произойдет вне зоны действия сотовой связи, сообщение о ней передано не будет. При использовании для таких целей системы C-V2X существует хоть и относительно низкая (учитывая то, что речь идет о труднодоступной местности), но

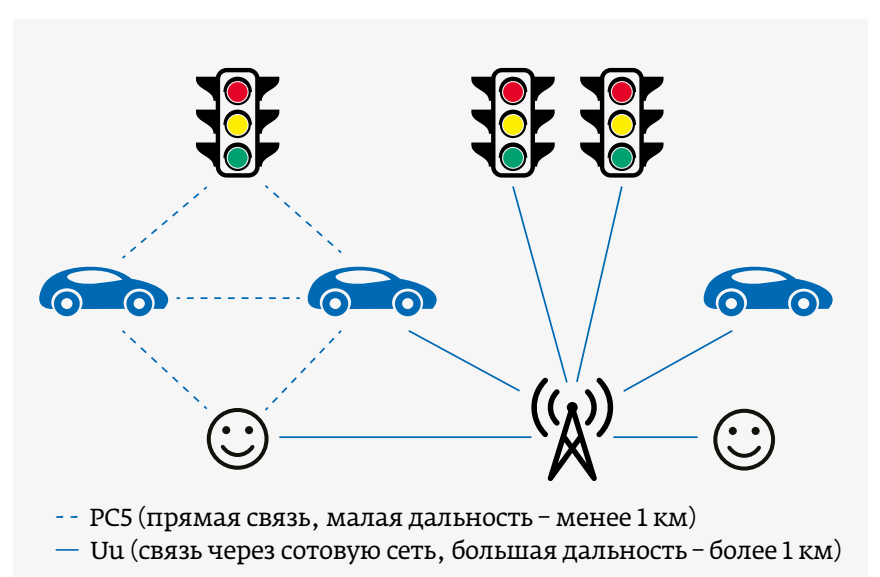

Рис. 1. Режимы взаимодействия автомобиля с внешней средой в системе C-V2X

не нулевая вероятность, что сообщение будет передано по цепочке из нескольких проезжающих автомобилей, что может оказаться принципиальным, когда речь идет о человеческих жизнях. В более вероятных сценариях прямая связь между транспортными средствами позволяет более быстро и надежно получать сведения от других автомобилей о различных дорожных ситуациях, находящихся за пределами видимости водителя, предупреждать аварии и т. п.

Еще одной неотъемлемой частью систем коммуникации автомобиля с внешней средой являются получение информации о местоположении и синхронизация, которые в C-V2X реализуются с помощью сервисов глобальных навигационных спутниковых систем (ГНСС).

Несмотря на то, что для применения стандарта C-V2X могут использоваться существующие сотовые сети, с его полноценным внедрением связан ряд проблем, одной из которых является то, что в этом случае резко увеличится количество абонентов, с чем сеть может не справиться. По этой и другим причинам начало широкого использования C-V2X ожидается с вводом в эксплуатацию сетей пятого поколения - 5G. Однако на данный момент релиз 16 стандарта 3GPP еще не принят: его утверждение сместилось примерно на девять месяцев относительно первоначального плана и в настоящее время ожидается в 2020 году. Это является основным недостатком стандарта C-V2X. Тем не менее европейские страны в большей степени ориентированы на него, и, вероятно, для России он также будет представлять более высокий интерес, чем DSRC.

C полноценным развертыванием систем V2X связаны и другие сложности вне зависимости от того, о каком стандарте идет речь. Учитывая то, что данные системы будут оказывать непосредственное влияние на безопасность дорожного движения, остро встает вопрос 
о подтверждении требований безопасности при развитии стандартов. Как уже говорилось, крайне важным фактором является кибербезопасность.

Поскольку транспортные средства могут использоваться в разных странах, необходимо обеспечение соответствия международным и региональным требованиям. Также должна быть обеспечена взаимозаменяемость модулей, так как системы могут строиться на аппаратуре различных производителей.

Еще одной особенностью систем V2X является необходимость их бесперебойной работы в условиях интерференции, в особенности в городских условиях, где радиочастотная обстановка достаточно сложна и дополнительно усугубляется переотражениями от зданий и других объектов.

Ассоциацией 5GAA и регламентирующими организациями ожидается, что список сложностей развертывания систем V2X будет расширяться, однако обозначенные проблемы должны уже сейчас учитываться в системах тестирования V2X.

На уровне приложений применение стандартов C-V2X и DSRC не будет иметь значительных различий. Иными словами, с точки зрения пользователя не должно иметь значения, на каком стандарте построена коммуникация между его автомобилем и внешней средой: он будет получать те же услуги, ту же информацию и сервисы примерно так же, как сейчас происходит с навигацией, используется ли приложением система ГлОНАСС или GPS. Поэтому и задачи, решаемые средствами контроля и измерений для систем V2X различных стандартов, в целом одинаковые. В то же время на уровне протоколов и структуры сети между стандартами существуют существенные отличия, и конкретные решения для тестирования также будут различаться. Рассмотрим основные особенности тестирования систем C-V2X, а также некоторые решения, которые для этой цели предлагает компания Keysight.

К основным измеряемым параметрам при тестировании систем C-V2X относятся:

- задержка;

- надежность передачи (коэффициент ошибок пакетов - PER);

- помехи и совместимость;

- дальность действия (чувствительность);

- адаптация кзагруженности сети;

- максимальные относительные скорости транспортных средств;

- динамические искажения в каналах;

- пропускная способность;

- точность определения координат с помощьюГНСС;

- функциональная совместимость (согласованность);

- безопасность канала;

- характеристики антенн и проч.
Для реализации тестирования системы необходимо три решения:

- рч-тестирование всей цепочки (end-to-end) - включает высокочастотные тесты, проверки протоколов, тестирование стека и функциональный контроль;

- эмуляция ГНСС для синхронизации по месту и времени;

- верификация и отладка.

В условиях отсутствия принятого стандарта C-V2X ceрийное производство таких систем невозможно, поэтому решения для контроля и измерений, которые на данный момент предлагает компания Keysight для C-V2X, ориентированы преимущественно на исследования и разработку. В соответствии с указанными выше необходимыми решениями для тестирования, они включают следующие три ключевых аппаратно-программных блока:

1. Платформа тестирования беспроводных устройств сети 5G UXM E7515B обеспечивает выполнение сигнальных РЧ-тестов (тестов с эмуляцией сети) и поддерживает стандарт 5G NR в субшестигигагерцовом диапазоне, а также в миллиметровом диапазоне с использованием внешнего оборудования.

2. Векторный генератор В4-сигнапов МХG N5182B используется для эмуляции служб ГНСС для позиционирования и синхронизации, формируя в реальном времени сигналы от нескольких спутников GPS, ГЛОНАСС, Beidou (Compass), QZSS и Galileo, при этом позволяя добавлять искажения сигналов различных типов.

3. Анализаторы спектра, приложения тестирования C-V2X, измерительные приложения серии $X$ и приложения тестирования стека интелпектуальной транспортной системы (ITS), с помощью которых выполняются несигнальные тесты (без регистрации в сети) и верификация на физическом уровне.

Рассмотрим подробнее сигнальное РЧ-тестирование C-V2X. В отличие от сигнальных тестов устройств сотовой связи в данном тестировании выполняется не только регистрация и проверка работы устройства в эмулированной сети, но также и эмуляция среды, включая другие транспортные средства, пешеходов, дорожные события и т. п. На рис. 2 показана возможная тестовая конфигурация. Тестируемый модуль подключается к эмулятору сети и эмулятору навигационного сигнала, после чего запускается определенный сценарий, обеспечивающий функциональный контроль устройства. Примером сценария может служить движение с предупреждением об аварии на дороге. При этом может быть эмулирована выдача предупреждения как от базовой станции (по каналу Uu), так и от другого транспортного средства (по каналу РС5).

Как уже было сказано, для несигнальных тестов на этапе исследований и разработки применяются анализаторы спектра и программные средства. До утверждения 


\section{Настроены, поверены и готовы к работе}

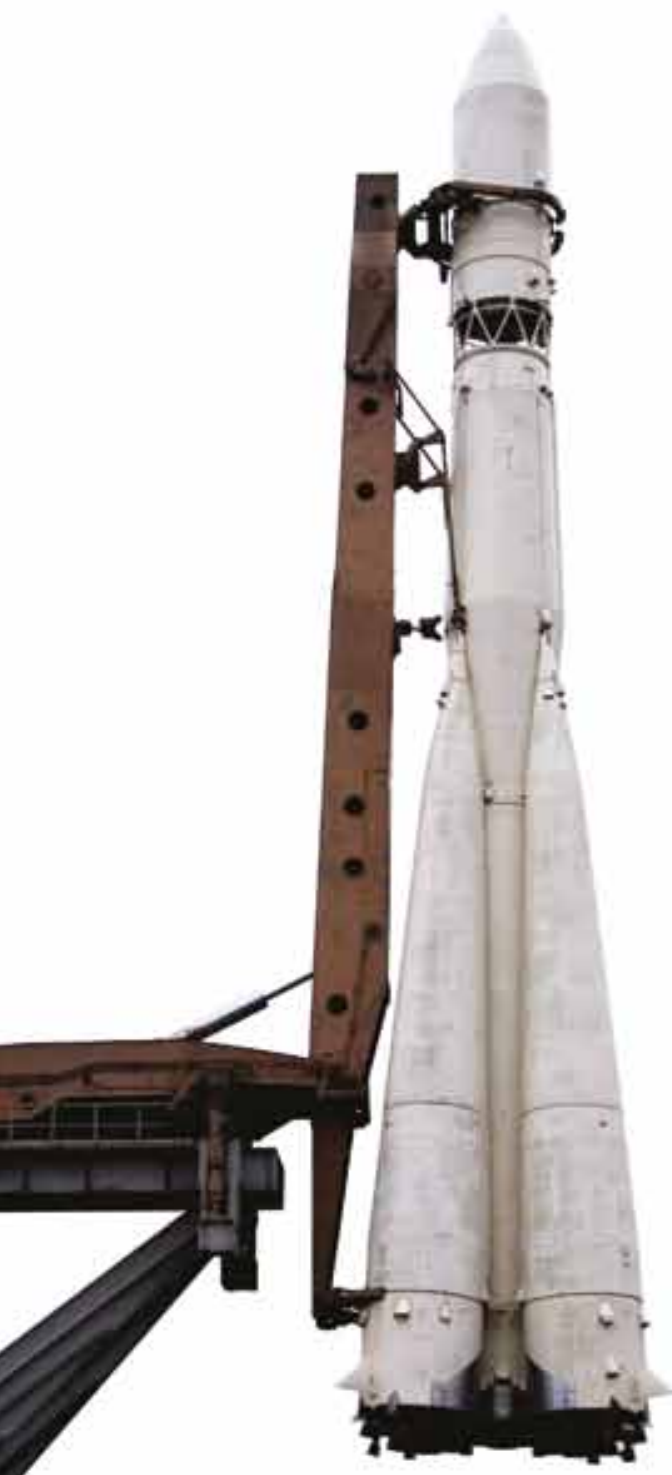

Подробнее: www.keysight.com/find/Poverka Тел.: 88005009286
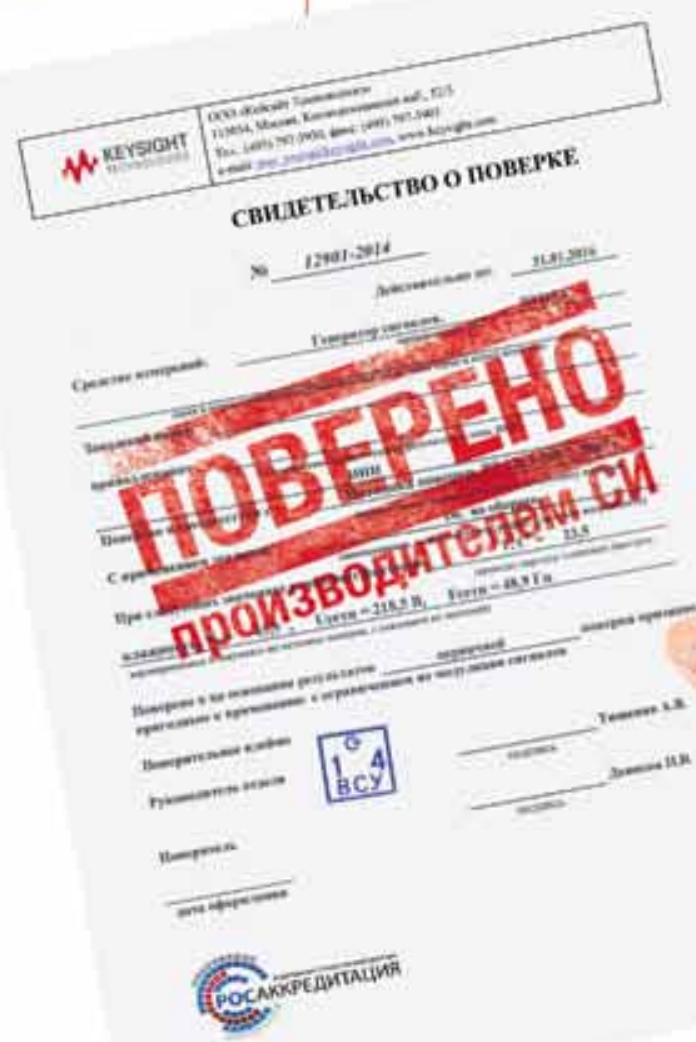

Ваши технические системы, критически важные для решения ответственных задач, должны обеспечивать безотказную работу в любых усповиях. Именно поэтому сервисный центр Keysight попучил акхредитацию на право поверки средств измерений и готов обеспечивать техническое обспуживание приборов под марками Keysight, Agilent $и$ HP.

Оригинапьные запчасти, автоматизированные тестовые системы, программное обеспечение для проведения калибровки и настройки, олытный персонал - все это позволяет выпопнять весь набор тестов в соответствии с требованиями завода-изготовителя максимально качественно и в сжатые сроки. Будьте уверены в точности ваших измерений! 
Векторный генератор ВЧ-сигналов MXС N5182B

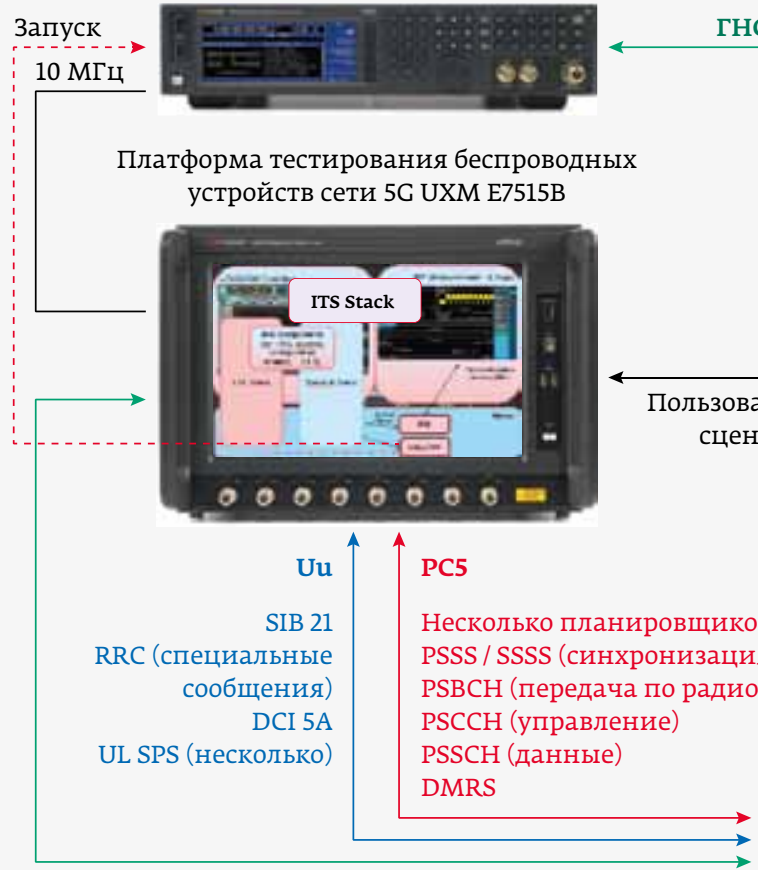

Последовательный интерфейс (АТ-команды)
Временная привязка

Синхронизация

Позиционирование

Траектория (в динамике)

Генерация и мониторинг сценариев интеллектуальной транспортной системы
Рис. 2.

Конфигурация для сигнального тестирования модуля $\mathrm{C}-\mathrm{V} 2 \mathrm{X}$
Тестируемое

транспортное средство редакции 16 стандарта 3GРР эти тесты основываются на стандарте редакции 14.

Хотя до запуска серийного производства модулей C-V2X еще существует некоторый временной зазор, стоит упомянуть о решении Keysight для несигнальных ВЧ-тестов абонентских устройств при их производстве, которое доступно уже сейчас. Это комплект для тестирования средств беспроводной связи ЕХM E6640A, который содержит в своем составе и генератор, и анализатор сигналов. Благодаря поддержке широкого спектра стандартов и многопортовости (в частности, при использовании многопортового адаптера имеется возможность подключения до 32 тестируемых устройств) данный комплект позволяет оптимизировать временные затраты при производственном тестировании.

До сих пор говорилось о тестировании в субшестигигагерцовом диапазоне. Однако предполагается, что при развертывании системы C-V2X на основе сетей 5G будет также использоваться и миллиметровый диапазон, с чем связан ряд новых проблем.

Одна из них заключается в невозможности выполнения измерений при очень высоких частотах через коаксиальный кабель. Это потребует применения в тестовых установках безэховых (или полубезэховых) камер и организации беспроводного тестирования ("по воздуху»), для чего Keysight может предложить ряд решений.

Другая проблема вызвана использованием направленного луча базовой станции, что сложно в реализации как на аппаратном уровне, так и на программном, поскольку устройства должны определять, когда на них направлен луч, а базовая станция должна направлять луч в соответствии с определенным алгоритмом на основе текущей обстановки. Например, если на некотором участке образовалось скопление автомобилей, базовая станция в этом направлении должна обеспечивать передачу большего объема данных и направлять лучи соответствующим образом, а при изменении дорожной обстановки менять направление лучей. Это требует новых видов тестирования. Такие тесты также уже реализованы Keysight в решениях с применением эмулятора каналов Propsim.

Говоря в целом о тестировании систем V2X, преимуществом компании Keysight является то, что она способна предоставить полный комплекс решений, охватывающих все уровни инфраструктуры сети, включая компоненты и чипсеты, абонентские устройства, базовые станции, дата-центры и корпоративный уровень, и позволяющих выполнять тестирование как аппаратуры, так и программного обеспечения. Это стало возможным, в частности, благодаря приобретению Keysight компаний Ixia и Anite.

Компания Keysight плотно взаимодействует с различными научными центрами, ассоциациями, компаниями автомобильной и телекоммуникационной отраслей и в настоящее время предлагает наиболее широкое портфолио решений для всех этапов жизненного цикла изделий для различных инженерных задач в области V2X. 


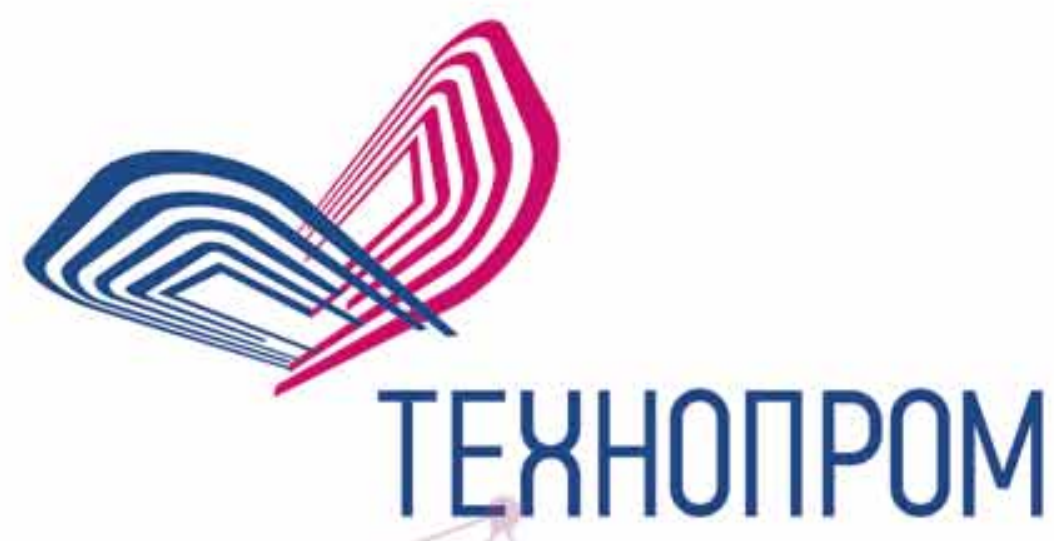

VII МЕЖДУНАРОДНЫЙ ФОРУМ И ВЫСТАВКА ТЕХНОЛОГИЧЕСКОГО РАЗВИТИЯ

\section{«ТЕХНОПРОМ-2019»}

\section{8-20 СЕНТЯБРЯ 2019}

МВК «НОВОСИБИРСК ЭКСПОЦЕНТР»

Организатор Оператор

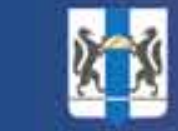

Правитепьство

Новосибирской области

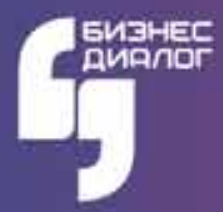

$8(800) 333-54-63$ звонок по России бесплатный forumtechnoprom.com 


\section{МИКРОСХЕМЫ СТАБИЛИЗАТОРОВ НАПРЯЖЕНИЯ \\ КАТЕГОРИИ КАЧЕСТВА «ВП» 1344ЕН}

Окончена ОКР «Генератор-5», в рамках которой разработаны микросхемы 1344ЕН1.8У, 1344ЕН2.5У и 1344ЕН3.3У категории качества «ВП». Микросхемы являются стабилизаторами напряжения (с низким остаточным напряжением) с фиксированными выходными напряжениями положительной полярности, со встроенным электронным ключом. Внутренний ключ управляется напряжением ТТЛ или КМОП-логики. ИМС находится во включенном состоянии, когда вход управления CONTROL в состоянии «логическая единица». Для уменьшения уровня выходного шума можно подключить внешний конденсатор к выводу коррекции шума.

Микросхемы предназначены для применения в малогабаритных кварцевых генераторах, а также источниках питания радиоэлектронной аппаратуры специального назначения.

Микросхемы изготавливаются в металлокерамическом корпусе типа 5221.6-1 и функционируют при температуре среды от -60 до $+125^{\circ} \mathrm{C}$.

Функциональные аналоги микросхем 1344ЕН1.8У, 1344ЕН2.5У и 1344ЕН3.3У - соответственно микросхемы TK71718S, TK71725S, TK717338S компании TOKO (Япония).

Технические условия - АЕНВ.431420.535 ТУ.

Габаритный чертеж микросхем серии 1344 в корпусе 5221.6-1

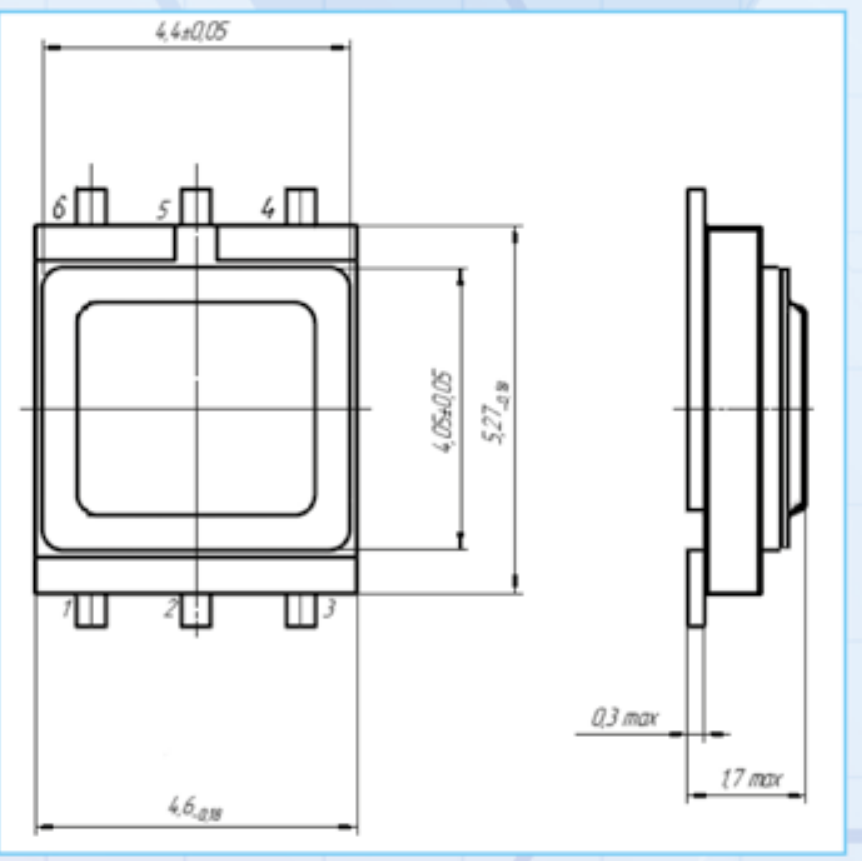

Назначение выводов

\begin{tabular}{|c|c|c|}
\hline Номер вывода & Обозначение & Назначение \\
\hline 1 & UIN & Вход \\
\hline 2,3 & UouT & Выход \\
\hline 4 & $\begin{array}{c}\text { NOISE } \\
\text { BYPASS }\end{array}$ & $\begin{array}{c}\text { Вывод } \\
\text { коррекции шума }\end{array}$ \\
\hline 5 & GND & Общий вывод \\
\hline 6 & CONTROL & Вход управления \\
\hline
\end{tabular}

Микросхемы 1344ЕН1.8У, 1344ЕН2.5У и 1344ЕН3.3У имеют стойкость к воздействию специальных факторов 7.И, 7.С и 7.К по ГОСТ РВ 20.39.414.2 с характеристиками 7.И6 - 5Ус; 7.И $-2 \times 4 \mathrm{~V}_{\mathrm{c}} ; 7 . \mathrm{C}_{4}-1 \times 5 \mathrm{У}_{\mathrm{c}}, 7 . \mathrm{K}_{1}-10 \times 1 \mathrm{~K}$;

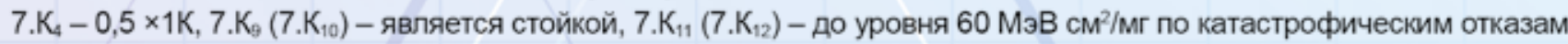
и тиристорному эффекту.

Поданы предложения для включения микросхем 1344ЕН1.8У, 1344ЕН2.5У и 1344ЕН3.3У в перечень ЭКБ 02. Возможна передача образцов микросхем 1344ЕН1.8У, 1344ЕН2.5У и 1344ЕН3.3У заинтересованным в их применении предприятиям для проведения тестирования.

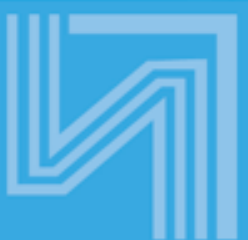

ОАО «ИНТЕГРАЛ» - 


\section{鸟 RHTEГPAR}

Табпица 1. Эпектрические параметры микросхем при приемке и поставке

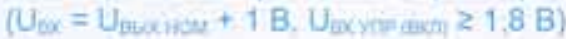

\begin{tabular}{|c|c|c|c|c|c|}
\hline \multirow{2}{*}{$\begin{array}{l}\text { Усповное } \\
\text { обозначение } \\
\text { микросхемы }\end{array}$} & \multirow{2}{*}{$\begin{array}{c}\text { Намменование параметра, } \\
\text { единица измерения } \\
\text { режим измерения }\end{array}$} & \multirow{2}{*}{$\begin{array}{l}\text { Eуквенное } \\
\text { обозначение } \\
\text { параметра }\end{array}$} & \multicolumn{2}{|c|}{ Hopma napamerpa: } & \multirow{2}{*}{$\begin{array}{l}\text { Temneparypa } \\
\text { kopnyca, } \mathrm{C}\end{array}$} \\
\hline & & & не менев & He 6 onee & \\
\hline 1344EH1.8Y & \multirow{3}{*}{$\begin{array}{l}\text { Выходное напряжение, В } \\
\text { при Uвх = Uвыхном }+1 \text { B, } \\
\text { Івых }=-5 \text { мA }\end{array}$} & \multirow{3}{*}{ Uabx } & 1,764 & 1.836 & \multirow{18}{*}{$25 \pm 10$} \\
\hline $1344 \mathrm{EH} 2.5 \mathrm{Y}$ & & & 2,462 & 2,538 & \\
\hline $1344 \mathrm{EH} 3.3 \mathrm{Y}$ & & & 3,250 & 3,350 & \\
\hline 1344EH1.8Y & \multirow{3}{*}{ 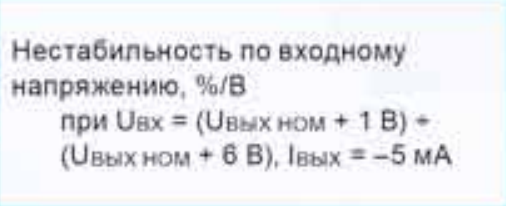 } & \multirow{3}{*}{ Ku } & - & 0,056 & \\
\hline $1344 \mathrm{EH} 2.5 \mathrm{Y}$ & & & - & 0.040 & \\
\hline $1344 \mathrm{EH} 3.3 \mathrm{Y}$ & & & - & 0,030 & \\
\hline \multirow{2}{*}{$\begin{array}{l}\text { 1344EH1.8Y, } \\
1344 \mathrm{EH} 2.5 \mathrm{Y} \\
1344 \mathrm{EH} 3.3 \mathrm{Y}\end{array}$} & $\begin{array}{l}\text { Нестабильность по току нагрузки, \%/А } \\
\text { при }-5 \text { мA } \leq \text { І І ы } \leq-75 \mathrm{MA}\end{array}$ & \multirow[t]{2}{*}{$\mathrm{KI}$} & - & 11,43 & \\
\hline & $n p и-5$ мA $\leq$ /выx $\leq-150$ mA & & - & 14,02 & \\
\hline \multirow{3}{*}{$\begin{array}{l}1344 \mathrm{EH} 1.8 \mathrm{Y} \\
1344 \mathrm{EH} 2.5 \mathrm{Y} \\
1344 \mathrm{EH} 3,3 \mathrm{Y}\end{array}$} & $\begin{array}{l}\text { Минимальное падение напряжения, В } \\
\text { при Івых }=-38 \mathrm{MA}\end{array}$ & \multirow{3}{*}{ Una min } & \multirow{3}{*}{-} & 138 & \\
\hline & 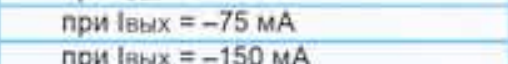 & & & 215 & \\
\hline & при Івых =-150 МА & & & 330 & \\
\hline \multirow{2}{*}{$\begin{array}{l}1344 \mathrm{EH} 1.8 \mathrm{y} \\
1344 \mathrm{EH} 2.5 \mathrm{Y} \\
1344 \mathrm{EH} 3.3 \mathrm{Y}\end{array}$} & $\begin{array}{c}\text { Tок потребления, MA } \\
\text { при Іеых }=0 \text { MA }\end{array}$ & \multirow{2}{*}{ Inot: } & - & 0,11 & \\
\hline & $\pi p и l_{8 b x}=-50 \mathrm{MA}$ & & - & 1,5 & \\
\hline $\begin{array}{l}1344 \mathrm{EH} 1.8 \mathrm{y} \\
1344 \mathrm{EH} 2.5 \mathrm{Y} \\
1344 \mathrm{EH} 3.3 \mathrm{y}\end{array}$ & 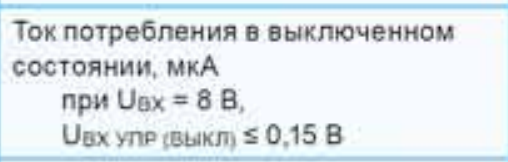 & Inot выKл & - & 0,1 & \\
\hline 1344EH1.8Y & $\begin{array}{l}\text { Tок разряда, MA } \\
\text { при } \cup_{\text {ogp }}=1,8 \text { B }\end{array}$ & \multirow{3}{*}{ IPAB } & 11 & - & \\
\hline 1344EH2.5Y & при UоБp $=2,58$ & & 18 & - & \\
\hline $1344 E H 3.3 Y$ & при Uовр $=3.3 \mathrm{~B}$ & & 23 & - & \\
\hline \multirow{2}{*}{$\begin{array}{l}1344 \mathrm{EH} 1.8 \mathrm{y} \\
1344 \mathrm{EH} 2.5 \mathrm{y} \\
1344 \mathrm{EH} 3,3 \mathrm{y}\end{array}$} & $\begin{array}{l}\text { Температурный коэффициент } \\
\text { напряжения. \%/ }{ }^{\circ} \mathrm{C} \\
\text { при Івых }=-5 \text { мA }\end{array}$ & aU & - & 0.03 & \\
\hline & $\begin{array}{l}\text { Дрейф выходного напряжения, \% } \\
\text { при Івых }=-5 \text { мА }\end{array}$ & $\Delta U_{\text {ebax }}$ & - & 1.5 & 125 \\
\hline \multicolumn{6}{|c|}{ Параметры входа управления CONTROL } \\
\hline \multirow{3}{*}{$\begin{array}{l}\text { 1344EH1.8Y, } \\
1344 \mathrm{EH} 2.5 \mathrm{Y} \\
1344 \mathrm{EH} 3.3 \mathrm{Y}\end{array}$} & $\begin{array}{l}\text { Ток по входу управления, МКА } \\
\text { Uвхупр }=1.8 \text { В. } \\
\text { состояние квкпючено }\end{array}$ & IBXYnP & - & 2.5 & \multirow{3}{*}{$25 \pm 10$} \\
\hline & $\begin{array}{l}\text { Напряжение включения на } \\
\text { входе управления, В, } \\
\text { состояние "включено» }\end{array}$ & U日X YחP (ВКЛ) & - & 1.6 & \\
\hline & $\begin{array}{l}\text { Напряжение выключения } \\
\text { на входе управления, В, } \\
\text { состояние «выкпюченож }\end{array}$ & Uax yпр (выкл) & 0.6 & - & \\
\hline
\end{tabular}

